\title{
Civil Justice in China
}

Representation and Practice in the Qing 
